\title{
Représentations du savoir féminin dans l'Espagne du Siècle d'or : \\ l'érudite, l'intellectuelle et la femme d'esprit dans les nouvelles d'Alonso de Castillo Solórzano
}

\author{
Christelle Grouzis-Demory \\ Université Paul-Valéry Montpellier
}

Cette étude se propose d'analyser les représentations du savoir féminin dans un corpus de nouvelles écrites par Alonso de Castillo Solórzano (1584-1648), publiées entre 1625 et 1649. Poète, dramaturge, historien, cet homme de lettres fécond de l'Espagne du Siècle d'or est le nouvelliste le plus prolifique sous le règne de Philippe IV. 
Le choix d'étudier le réseau d'images induites par les figures féminines du savoir dans ses récits de fiction s'explique par la place importante et singulière qu'elles occupent aussi bien dans les nouvelles que dans le récit-cadre des œuvres dans lesquelles elles sont insérées. En outre, les textes de Castillo Solórzano élaborent des représentations du savoir féminin particulièrement riches et variées (Grouzis Demory, p. 198222). Aux côtés de la femme érudite, apparaissent d'autres figures telles que l'intellectuelle et la femme d'esprit.

S'interroger sur ces images féminines, c'est aussi s'intéresser à l'accès des femmes fictives à la culture profane. Qui leur délivre le savoir? Dans quelles conditions est-il transmis? À quelle catégorie sociale appartiennent ces personnages féminins qui investissent un domaine traditionnellement masculin?

Les femmes «savantes ${ }^{1}$ » étant souvent la cible de critiques ou de railleries en Espagne au Siècle d'or, il conviendra de s'interroger sur l'image positive et valorisante que nous en livrent les nouvelles de Castillo Solórzano.

\section{Représentations du savoir féminin}

Les figures de l'érudite, de l'intellectuelle et de la femme d'esprit constituent des motifs récurrents dans la production nouvellistique de Castillo Solórzano. Par le terme "érudite » (du latin eruditus, du verbe erudire, instruire), nous entendons la femme docte et bien instruite qui a des lettres, de la culture,

\footnotetext{
1 Nous entendons par le terme « savante » la femme cultivée, lettrée et érudite mais aussi celle qui est très habile et qui s'y connaît dans son art, sa spécialité.
} 
du savoir, mais aussi celle qui est tout particulièrement versée dans les matières d'érudition et de sciences.

C'est le cas par exemple de la protagoniste mise en scène dans la nouvelle intitulée « La injusta ley derogada » (Fiestas del jardín). Arminda reçoit en effet une instruction scientifique d'ordinaire réservée aux hommes et se montre experte en plusieurs langues. De même, dans "La inclinación española " (La Quinta de Laura), les infantes de Pologne sont décrites comme étant des femmes cultivées, versées dans les langues qu'elles ont longuement étudiées. L'érudition féminine est aussi mise en avant à travers les personnages féminins des récitscadres. Le personnage éponyme de Los Alivios de Casandra en est un exemple éclairant. Polyglotte, la jeune femme maîtrise à la perfection le latin : «supo perfectamente algunas lenguas, y en particular la latina ${ }^{2} »$.

Cette représentation de l'érudition féminine dans l'œuvre de Castillo est d'autant plus singulière qu'elle est peu répandue dans les nouvelles espagnoles du XVIIe siècle 3 .

Aux côtés de la femme érudite, l'auteur accorde une place primordiale à l'intellectuelle et à la femme d'esprit. Si ces deux

\footnotetext{
2 Je traduis : « elle maîtrisa certaines langues à la perfection, en particulier le latin».

3 La lecture d'un grand nombre de nouvelles espagnoles post-cervantines nous a révélé que la figure de la femme érudite était peu souvent représentée. On la retrouve chez Juan de Piña, qui lui accorde une place importante dans la nouvelle "Los amantes sin terceros», où Feliciana, fervente admiratrice d'Ovide, est présentée comme étant versée dans la poésie latine. Dans ce même récit, doña Antonia, experte en philosophie, en mathématiques et dans les arts libéraux, est comparée à la célèbre Juliana Morella (Piña, 1624, p. 4142). L'érudite apparaît en outre dans le récit de Céspedes y Meneses intitulé "El desdén del alameda», qui met en scène une jeune femme lettrée, maîtrisant le latin et la rhétorique (1980 [1623], p. 115).
} 
figures féminines du savoir sont extrêmement proches, nous avons fait le choix de les différencier en circonscrivant la figure de l'intellectuelle, c'est-à-dire celle « qui a un goût prononcé (ou excessif) pour les choses de l'intelligence, de l'esprit » et « dont la vie est consacrée aux activités intellectuelles », à l'écrivaine, la poète, l'amatrice éclairée de littérature (théâtre, prose, poésie). Les occurrences ne manquent pas dans les récits de Castillo Solórzano.

Ainsi, les femmes de la fiction se piquent d'aimer le théâtre et les vers. Doña Emerenciana, dans « Las dos dichas sin pensar» (Noches de placer), célèbre en amatrice éclairée de théâtre l'ingéniosité de don Diego Jiménez de Enciso, auteur de la pièce La Mayor hazaña de Carlos Quinto, qu'elle voit en compagnie d'une amie. À travers le personnage d'Emerenciana, la nouvelle nous éclaire sur l'engouement que suscitaient à l'époque les comedias chez les femmes nobles.

Par ailleurs, les personnages féminins montrent une finesse d'appréciation poétique. Si elles sont majoritairement sensibles à la poésie galante, elles affectionnent aussi la poésie satirique. C'est le cas, par exemple, de Gerarda et de Laudomia, qui saluent le talent de poète du gentilhomme don Fernando dans «Amor con amor se paga» (Los Alivios de Casandra). Dans «Quien todo lo quiere, todo lo pierde» (La Garduña de Sevilla), doña Isabel fait l'éloge de la composition poétique que son prétendant lui écrit. En fine connaisseuse, elle commente l'écriture des vers. Rien d'étonnant lorsque l'on sait que la jeune femme est experte en la matière. On apprend en effet dans le récit qu'elle est célébrée par la haute noblesse valencienne pour ses grandes qualités de poète, son esprit créatif et sa sagacité. 
La femme poète est largement représentée dans les nouvelles. Dans « La inclinación española » (La Quinta de Laura), l'infante de Pologne, nous dit-on, savait composer de magnifiques vers. Dans «El amor con amor se paga» (Los Alivios de Casandra), Lucrecia rapporte à propos de Gerarda qu'une autre de ses qualités était d'écrire de jolis poèmes. Il faut remarquer que la poésie qu'elle fait parvenir à don Fernando le plonge dans le plus grand trouble. Son écuyer, don Garcerán, ne cache pas non plus son admiration pour son talent de poète inégalable, comme ne manque jamais de le souligner le narrateur: " ¿en la poesía quien la excede? Tan galantes versos hace, que es admiración de todos cuantos se precian de hacerlos ${ }^{4} »$ (Castillo Solórzano, 1640, p. 99). Les différentes compositions poétiques écrites, lues ou chantées par les femmes s'inscrivent dans la grande tradition de la poésie lyrique qui exalte la beauté de l'aimée, l'amour irrésistible, les émois amoureux, l'absence de la personne aimée ou encore la réciprocité du sentiment amoureux.

La femme poète est aussi présente parmi les personnages du récit-cadre de Los Alivios de Casandra. La fille du marquis Ludovico manie le vers, et ce, dans plusieurs langues, parmi lesquelles le latin, l'italien et l'espagnol. Enfin, il est intéressant de souligner l'évocation, au sein même de la fiction, de femmes poètes issues de la réalité extra-textuelle, comme nous le relevons dans La Garduña de Sevilla. Le personnage de Monsalve y fait l'éloge en ces termes de la poète andalouse Ana Caro de Mallén, contemporaine de Castillo Solórzano : «con sus

4 Je traduis: «En poésie, qui la surpasse? Ses vers sont si beaux qu'ils suscitent l'admiration de tous ceux qui se targuent d'en écrire ». 
dulces y bien pensados versos suspende y deleita a quien los oye $y$ lee 5 » (Castillo Solórzano, 1642, p. 67).

L'amatrice éclairée de poésie et la femme poète ne sont pas les seules représentations qui nous sont données de l'intellectuelle. D'autres occurrences fort nombreuses de femmes réceptrices ou productrices de nouvelles jalonnent l'œuvre de Castillo Solórzano. Dans La Garduña de Sevilla, Rufina, lectrice assidue de ce type de récits brefs et particulièrement sensible au style littéraire, implore Monsalve de lire l'une de ses nouvelles extraites de son recueil Camino divertido. De nombreuses remarques ayant trait à l'art d'écrire des nouvelles émanent de protagonistes femmes cultivées initiées au genre de la nouvelle italienne et espagnole. Il est intéressant de souligner que les allusions à des pratiques littéraires reprenant certaines recommandations issues de textes théoriques (importance du divertissement, double finalité à la fois récréative et édifiante) sont très souvent prises en charge par les personnages féminins.

Aux côtés de ces femmes amatrices éclairées et réceptrices de nouvelles, nous relevons des exemples de femmes auteurs. Dans La Quinta de Laura, Artemidora rapporte de mémoire la nouvelle "El desdén vuelto favor», qu'elle a ellemême écrite. La singularité de ce texte, qui réside dans l'absence de l'emploi de la lettre i, est applaudie par Laura et les autres dames de compagnie. Soulignons ici la mise en abyme entre le personnage fictif d'Artemidora, auteur de la nouvelle "El desdén vuelto favor», et les nouvellistes espagnols du XVIIe siècle, qui eurent aussi recours à la même stratégie

5 Je traduis : « ses vers agréables et bien pensés enchantent et ravissent celui qui les entend et les lit». 
d'écriture dans le but d'innover et de surprendre leur public ${ }^{6}$. Et si dans la fiction, c'est une femme qui écrit, dans la réalité, il s'agit d'un homme (Castillo Solórzano), lequel, derrière la figure du narrateur, reconnaît le talent de l'écrivaine et ses qualités d'écriture.

Enfin, la femme d'esprit occupe une place tout aussi importante que l'intellectuelle dans les écrits de Castillo Solórzano. Nous entendons par "femme d'esprit», la femme spirituelle dotée de vivacité d'esprit et d'ingéniosité, celle qui a de l'à-propos, qui sait briller et plaire. Cette figure apparaît notamment dans Noches de Placer à travers le personnage de doña Camila, dont les qualités exceptionnelles d'oratrice suscitent l'engouement de l'auditoire. Le narrateur ne manque d'ailleurs jamais de souligner dans le récit sa parfaite maîtrise de l'art de conter et son ingéniosité au moment de rapporter la nouvelle intitulée « El celoso hasta la muerte».

Dans Tardes entretenidas, les personnages féminins, en plus de raconter des histoires et de réciter des poèmes, proposent à leurs interlocuteurs une énigme en vers. L'énigme constitue une autre activité de l'esprit à laquelle s'adonnent avec brio les femmes de la fiction. Par le truchement de ce loisir, les facultés intellectuelles féminines sont constamment mises en avant. La répétition des substantifs ingenio, entendimiento, discreción et leurs dérivés sont autant d'éléments lexicaux récurrents constitutifs des portraits de ces femmes d'esprit. Rappelons que dans l'Espagne du Siècle d'or, le mot ingenio

\footnotetext{
6 On citera par exemple les nouvelles d'Alonso Alcalá y Herrera : "Los dos soles de Toledo", écrite sans la lettre a, "La carroza con las damas, sin la

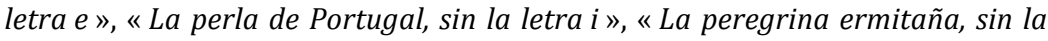
letra $o$ » et « La serrana de Sintra, sin la letra $i$ » (Alcalá y Herrera, 1641).
} 
renvoie aux qualités intellectuelles, qu'elles soient naturelles ou acquises, à l'entendement, à l'intelligence, à l'esprit. Il suppose presque toujours la subtilité, la finesse, l'ingéniosité, l'esprit créatif. En outre, la discreción désigne, selon le contexte, la sagesse, le bon sens, le discernement, la vivacité d'esprit et l'intelligence.

La grande majorité des figures féminines du savoir s'illustrent par ailleurs dans les arts d'agrément. Le chant, la musique, la danse sont autant de divertissements raffinés qui avaient cours dans les milieux de la noblesse et qui constituaient l'expression d'un monde policé. Rappelons que dans «Los efectos que hace el amor» (Los Alivios de Casandra), Diana évoque l'extrême habileté de la princesse Porcia à jouer du théorbe et de la harpe. Dans "La ingratitud castigada» (La Quinta de Laura), Costanza est décrite comme étant une musicienne exceptionnelle maîtrisant les instruments en usage aussi bien en Espagne qu'en Italie, parmi lesquels le théorbe, le clavecin, la guitare et le violon.

Enfin, pour compléter ce portrait de la femme versée dans les arts, nous évoquerons la figure atypique de Gerarda, qui excelle en peinture. L'héroïne se distingue plus particulièrement dans l'art du portrait et de l'autoportrait. Dans « Amor con amor se paga » (Los Alivios de Casandra), son étonnante virtuosité est sans cesse relayée dans le discours des personnages masculins, qui mettent en avant le réalisme édifiant de ses toiles.

\section{Portrait social de ces femmes savantes}

Les figures féminines du savoir que nous venons d'évoquer sont indissociables des catégories sociales supérieures auxquelles 
elles appartiennent. Cette situation n'a rien de surprenant, car à l'époque, l'accès au savoir était le privilège d'une élite. Souveraines, princesses, membres de l'aristocratie européenne ou encore jeunes femmes issues de la haute noblesse italienne et espagnole, les personnages féminins sont entourés des meilleurs précepteurs et d'éminents savants chargés de les éduquer et de les instruire. Le savoir est toujours transmis par des hommes dans le cadre du cercle familial (espace clos et privé) et les femmes n'y accèdent pas de manière autonome. Dans Los Alivios de Casandra, par exemple, l'héroïne incarne le modèle de la femme docte dont le savoir est délivré par les plus grands savants espagnols. De même, dans La Quinta de Laura, l'érudition de la jeune femme est le fait d'illustres maîtres.

Cependant, le savoir féminin ne se limite pas exclusivement aux classes favorisées dans les nouvelles de Castillo Solórzano. Même si elles sont rares, certaines femmes lettrées sont issues du peuple. C'est le cas de Rufina, dans $L a$ Garduña de Sevilla, et de la servante de doña Luciana, dans «Elproteo de Madrid» (Tardes entretenidas). Le côté invraisemblable et rocambolesque de ces situations, justifié par la fiction, est l'un des ressorts employés par l'auteur, qui n'hésite pas à abandonner toute vraisemblance pour atteindre son principal objectif : divertir ses lecteurs.

\section{Défense du savoir féminin}

Les nouvelles de Castillo Solórzano reposent en grande partie sur la promotion d'un modèle de femme érudite, instruite, cultivée, intellectuelle et spirituelle. Dans les nouvelles de cet auteur, l'accès des femmes au savoir ne les conduit pas au vice, 
contrairement à la thèse des Anciens. Il ne représente pas non plus une menace ou un danger, idée communément admise dans la société espagnole de l'époque. Bien au contraire, ce savoir leur permet de s'illustrer dans un champ d'ordinaire réservé aux hommes. En ce sens, les images féminines déployées dans les textes s'écartent du modèle idéal de la femme passive, assujettie et réduite au silence promu par l'idéologie dominante masculine et religieuse en vigueur en Espagne au XVIIe siècle.

Cette représentation positive des savoirs féminins peut apparaître comme une stratégie d'écriture, de la part de l'auteur, visant à séduire un public composé majoritairement de femmes issues de la haute noblesse. Elle permet en effet de véhiculer l'image d'une société féminine intellectuellement supérieure et policée, miroir sans doute «idéalisé » dans lequel les lectrices se plaisent à se regarder.

Ces représentations féminines sont aussi sans doute à relier à la mission civilisatrice dévolue aux femmes dès la Renaissance et à leur rôle éminent dans la sociabilité mondaine. Castillo Solórzano paraît donc s'inscrire dans la lignée de Castiglione, qui défend dans Le Livre du Courtisan: "l'égale dignité des hommes et des femmes, capables de mêmes vertus de cœur et d'esprit » (Grell / Ramière de Fortanier, p. 10).

Les images féminines qui nous sont données et, plus particulièrement, celles de l'intellectuelle rendent peut-être compte, comme le suggère Fernando Copello, de l'évolution d'une perception de la femme dans l'Espagne du XVIIe siècle et de son rôle dans la vie intellectuelle et littéraire. Castillo Solórzano s'en ferait l'écho dans son œuvre à l'instar de Lope de Vega dans sa comédie en prose intitulée La Dorotea (1632). 
Par le biais de ses personnages, Castillo semble aussi légitimer la place des femmes dans les milieux lettrés en rendant notamment un hommage appuyé à des écrivaines contemporaines. Dans La Garduña de Sevilla, Monsalve ne louet-il pas le génie littéraire de María de Zayas, amie intime de l'auteur et qu'il avait l'habitude de côtoyer dans les salons littéraires? Au sujet des nouvelles qu'elle a composées, le personnage affirme :

son diez asombros para los que escriben deste género, pues la meditada prosa, el artificio dellas y los versos que interpola, es todo tan admirable, que acobarda las más valientes plumas de nuestra España ${ }^{7}$.

De cette manière, Monsalve participe à la promotion de l'œuvre de la poète et nouvelliste madrilène comme Castillo lui-même dans les textes préliminaires de Novelas amorosas y ejemplares (María de Zayas, 1637).

Sous la plume de Castillo, le savoir intellectuel n'est plus l'apanage des hommes, tel que le souligne en ces termes Monsalve :

es atrevimiento grande escribir en estos tiempos, cuando veo que tan lucidos ingenios sacan a luz partos tan admirables cuanto ingenioso y no sólo hombres que profesan saber humanidades ${ }^{8}$.

\footnotetext{
7 Je traduis : « ce sont dix merveilles pour ceux qui écrivent des nouvelles, car la prose bien pensée, la finesse de l'écriture et les vers qu'elle intercale sont si admirables qu'ils impressionnent les meilleures plumes de notre pays ».

8 Je traduis : « il est bien audacieux d'écrire à notre époque lorsque je vois que de si brillants esprits engendrent des œuvres aussi géniales qu'admirables, et pas seulement des hommes qui proclament leur connaissance des humanités ».
} 
La publication au XVIIe siècle d'œuvres écrites par des auteurs femmes ayant acquis une certaine reconnaissance permet d'en témoigner. Parmi les plus connues, citons Ana Caro Mallén, María de Zayas ou encore Mariana de Carvajal.

Enfin, cette réévaluation du rôle de la femme dans l'œuvre de Castillo est peut-être plus généralement à rapprocher des courants féminocentrique et égalitaire européens du XVII ${ }^{\mathrm{e}}$ siècle. 


\section{Bibliographie}

AlCAlÁ y Herrera, Alonso. (1641), Varios efectos de amor en cinco novelas ejemplares sin una de las cinco letras vocales, Lisbonne, Francisco de Costa.

Castillo SolOrzano, Alonso (de). (1634), Fiestas del jardín, Valence, Felipe Pincinali.

—. (1942 [1642]), La Garduña de Sevilla y anzuelo de las bolsas, Federico Ruiz Morcuende (éd.), Madrid, Espasa-Calpe, Clásicos Castellanos.

-. (1649), La Quinta de Laura, Saragosse, Matías de Lizau.

- (1640), Los Alivios de Casandra, Barcelone, Jayme Romeu.

-. (1906 [1631]), Noches de placer, Emilio Cotarelo y Mori (éd.), Madrid, Imprenta Ibérica, Colección selecta de antiguas novelas españolas.

—. (1992 [1625]), Tardes entretenidas, Patrizia Campana (éd.), Barcelone, Montesinos, Biblioteca de clásicos y raros.

Cespedes y Meneses, Gonzalo (de). (1980 [1623]), Historias peregrinas y ejemplares, Yves-René Fonquerne (éd.), Madrid, Clásicos Castalia.

GrelL, Chantal et Arnaud RAMiÈre DE Fortanier. (2004), $L^{\prime}$ Éducation des jeunes filles nobles en Europe XVII - XVII ${ }^{e}$ siècles, Paris, Presses de l'Université Paris-Sorbonne.

GrouZIS DEMORY, Christelle. (2011), Les Femmes, la famille, la vie affective en Espagne au XVII siècle: représentations littéraires et réalités sociales dans les nouvelles d'Alonso de 
Castillo Solórzano, Montpellier, Université Paul-Valéry Montpellier.

PIÑA, Juan (de). (1624), Novelas ejemplares y prodigiosas historias, Madrid, Juan González.

VEGA, Lope (de). (1632), La Dorotea, Madrid, Alonso Pérez.

ZAYAS y SOTOMAYOR, María (de). (1637), Novelas amorosas y ejemplares, Zaragoza, Pedro Esquer.

\section{Résumé}

Cette étude se propose d'analyser la représentation des savoirs féminins dans un corpus de nouvelles d'Alonso de Castillo Solórzano (1584-1648). Nous nous intéresserons tout d'abord aux différentes images de la femme érudite, de l'intellectuelle et de la femme d'esprit, avant de nous interroger sur l'accès de ces femmes fictives à la culture profane. Versées dans les sciences, les lettres, les langues et les arts d'agrément, écrivaines ou encore poètes, les héroïnes qui investissent un domaine d'ordinaire réservé aux hommes contribuent à véhiculer l'image valorisante de la femme lettrée.

\section{Abstract}

Our study intends to analyse the representation of women's knowledge in a corpus of Alonso de Castillo Solórzano's short stories. First of all, we will take an interest in the various images of the erudite woman, the intellectual and the woman of wit before studying the access these fictional female characters have to profane culture. By breaking into traditionally masculine fields, such as science, literature, languages or arts, these heroines, whether they be writers or poetesses, play a major role in conveying a gratifying image of the well-read woman. 